Magna Scientia Advanced Research and Reviews

eISSN: 2582-9394

Cross Ref DOI: $10.30574 / \mathrm{msarr}$

Journal homepage: https://magnascientiapub.com/journals/msarr/

(REVIEW ARTICLE)

\title{
Oncofetal genes and its association with bivalent chromatin and DNA methylation: (A Review)
}

\author{
Ayesha Shahid ${ }^{1, *}$ and Aquib Belal Khan ${ }^{2}$ \\ ${ }^{1}$ Department of Pharmacology, BIT Mesra. \\ ${ }^{2}$ Department of Pharmaceutical Chemistry, Jamia Hamdard.
}

Magna Scientia Advanced Research and Reviews, 2022, 04(01), 001-003

Publication history: Received on 11 December 2021; revised on 14 January 2022; accepted on 16 January 2022

Article DOI: https://doi.org/10.30574/msarr.2022.4.1.0021

\begin{abstract}
Oncofetal genes are primarily those genes that are expressed in fetal tissue but remain in a state of inactivation in adult. Assessing the various factors involved in reactivation of oncofetal gene, the predominant one that represent a distinct mechanism is bivalency. Evidence suggest that DNA methylation in context to bivalent domain of Histone 3 particularly at Lysine 4 and 27, serves as a major site for reactivation of silenced transcriptional activity along with protein complexes Tri-thorax (TrxG) and Poly-comb(PcG). The malignancy of proto- oncogene establishes a correlation with $\mathrm{CpG}$ rich DNA sequence that adds appropriate methylation providing rapid activation to express the silenced genes.
\end{abstract}

Keywords: Oncofetal genes; Methylation; Bivalency; Repression; Activation

\section{Introduction}

Oncofetal genes are those genes which are produced or expressed during development of fetus particularly but are also found in adult tissue in very low amount. These oncofetal genes are found to be suppressed in adult tissue but due to some retro genetic expression it is found to be expressed again in cells associated with tumor. There are many oncofetal genes that are discovered so far such as alpha fetoprotein (AFP), carcinoembryonic antigen (CEA), feto-sulfoglycoprotein (FSA), tissue polypeptide antigen (TPA) and pancreatic oncofetal antigen (POA)

Furthermore, oncofetal antigen when found in adult body can serve as a diagnostic marker in detection and identification of initial progression of oncogenesis [1]. The production of these antigen occur due to activation of certain genes that serve as control genes or by post translational modification.

Oncofetal gene expression, is typically a cause of cancer progression. There are many post transcriptional modification in the histone which governs the epigenesist mechanism leading to onco-genesis.

\section{Etiology behind Oncogenesis via bivalency}

Histone belongs to a family of protein that have a key role in providing structural role in DNA packing into cell. In order to be in a position to be fit inside nucleus, the DNA is wrapped around histone protein. It is then further made to participate in chromosome formation. They also are critical in gene regulation and expression [1]

Histone protein consist of four core histone H2A, H2B, H3 and H4 respectively which one combine forms nucleosome. These core proteins undergo many post-transcriptional modification such as acetylation, methylation, phosphorylation and butyrylation. The structure of histone protein consists of fold of $\mathrm{N}$ terminal tail and $\mathrm{C}$ terminal [2]. All modification

\footnotetext{
${ }^{*}$ Corresponding author: Ayesha Shahid

Department of Pharmacology, BIT Mesra.

Copyright (C) 2022 Author(s) retain the copyright of this article. This article is published under the terms of the Creative Commons Attribution Liscense 4.0.
} 
in histone core have important role in affecting structure of chromosome. Basically all changes occur in the amino acid of Arginine, Lysine, Serine and threonine. [3,4]

Methylation of histone refers to the process through which methyl group is added to the amino acid residue of core protein of histone. This is further proceeded to make nucleosome that wraps around DNA to form chromosome [5]. The methylation of DNA has a primary role in its activation and inactivation, in context to lysine. The residues of lysine at 4,9,27, and 36 are considered as various important sites for methylation to occur. Among such sites Histone3 Lysine4 (H3k4) and Histone 3Lysine 27(H3K27) are one of the evolutionary conserved site modification [6]. These essentially bears function for regulation of gene along with DNA repair as well as during cell division in transcriptionally state. Many methylations too occur such as mono-methylation, di-methylation, tri-methylation that acts as enhancer and are regarded as marker of regulatory genes [7].

Bivalent chromosomes have histone modification at the site where it has property of both activating and repressing. Histones that are methylated function [8] either as repressor or activator. Oncofetal proteins that are encoded by gene are observed to be marked by bivalent modification at histone or are not marked. But indeed their regulators are upregulated and are marked bivalently. It is observed that when combination of both activating and repressing gene are present at promoter region or enhancer region it leads to expression of genes at low level but these low concentrations are found to be highly rapid activating.

The tri-methylation of amino acid lysine 4 and 27 of histone confers a specific site representing peculiar marks regulating the bivalency. This is again under an influential and dominating role of two epigenetic factor that interplay in malignancy. These factors that mediate the modification occurring in chromatin are known as polycomb and trithorax group protein. Various research study reveal that these two protein complex work opposite to each other as well as helps in maintaining the chromatin structure in a state of "on and off" in phase of transcription. A minor alteration or an imbalance in mechanism of chromosome on and off renders activation of oncogenes thereby favoring cancer and tumorigenesis.

Tri-thorax (TrxG) , a complex involved in epigenetic regulation, belongs to those class of protein that facilitate activation of gene expression. This occurs basically when methylation takes place on Histone 3lysine 4.

Polycomb (PcG), another complex playing major role in context to epigenetic regulation. It is a unit of diverse associated factor incorporating vivid characteristic function. Initially it has a polycomb repressor complex 1 and 2 respectively where the later serve the function of establishing code of histone and the other does the function of interpretation. Further the mechanism reveals involvement of enhancer known as Zest Homolog 2(EZH2). This in turn is linked with SET domain, comprising of enzyme Histone methyl transferase that mediates tri-methylaion at histone 3Lysine 27, thereby causing inactivation of chromatin and repression of gene expression.

Similarly Trithorax (TrxG), also known as mixed lineage leukemia in mammals comprises of enhancer Zest homolog 2 (EZH2) along with SET domain enzyme histone methyl-transferase. This enzyme has the capacity to establish methylation at histone 3 lysine 4 , ultimately leading to activation of chromatin marks.

The overall consequence of bivalent gene exhibiting pathways of both activating and repressing gene at promoter site is found to be [9] blocked by polycomb repressive complex 1 (PRC1). Evidence reveals, PRC 1 blocks the promoter site through interruption at the RNA polymerase II, thereby facilitating inhibition of elongation of transcription. Thus the activation is found to get poised and often on slight stimulation occurs very rapidly.

Besides these, analysis throw light on the finding suggesting CpG sites as one of the predominant factor in precipitating cancer via hyper-methylation. The $\mathrm{CpG}$ promoter sequence are maintained in a state of un-methylation during embryogenesis. Finding suggests that bivalent chromatin divide themselves into H3K4 trimethylation or H3K2 7 trimethylation while being in state of embryogenesis [10]. DNA sequence rich in CpG when unmethylated become accessible to recruit H3K4 trimethylation more easily than H3k27 trimethylation. Such aberrant hyper-methylation represses the physiology of DNA repair and tumor suppression and often represents an established site for bivalent domain. Here comes the role of enzyme methyltransferase (DNMT3a) which, in the presence of H3K4 trimethylation provides anchorage to methylate DNA [11]. Thus, CpG promoter sites along with hyper methylation and bivalent marked are potential factors in silencing cancer where by allowing the spontaneous activation of such transcriptional reactivation leading to oncogenesis via oncofetal genes. 


\section{Conclusion}

Experimental evidence illuminates the significance of oncofetal genes to be used as tumor marker. The post transcriptional modifications along with bivalent chromatin present a prerequisite to target the methylation state and histone modifications. Therefore, largely focusing on the mechanistic approach which reactivate such proto-oncogene, depends on the enzyme involved including DNA methyl transferase and enhancer EZH2. Being the potential target in contributing to establishment of cancer, oncogenesis offers an initiative for development of various challenging therapeutic approach. Data analysis reveals that oncofetal genes as an architectural epigenetic mechanism in cancer progression and with emerging targeting methods it can provide a novel avenue in drug development.

\section{Compliance with ethical standards}

\section{Acknowledgments}

The completion of this review article could not have possible without the support of my co-author whose insightful knowledge and data analysis made this article a success.Last but not the least I would like to thank my parents without them it would not have been possible.

\section{Disclosure of conflict of interest}

I and my co-author have no conflict of interest associated with this publication and there has been no significant financial support for this work that could have influenced its outcome.As the main author, I confirm that the manuscript has been read and approved for submission by my co-author.

\section{References}

[1] Chu TM. Biochemical Markers for Cancer. New York, Marcel Dekker. 1982.

[2] Chi P, Allis CD, Wang GG. Covalent histone modifications-miswritten, misinterpreted and mis-erased in human cancers. Nat Rev Cancer. 2010; 10: 457-4.69.

[3] Cohen I, Poreba E, Kamieniarz K, Schneider R. Histone modifiers in cancer: friends or foes, Genes Cancer. 2011; 2: $631-647$.

[4] Morales V, Richard-Foy H. Role of histone N-terminal tails and their acetylation in nucleosome dynamics. Mol Cell Biol. 2000; 20: $7230-7237$.

[5] Eissenberg JC, Shilatifard A. Histone H3 lysine 4 (H3K4) methyl ation in development and differentiation. Dev Biol. 2010; 339: 240 -249.

[6] Shilatifard A. Molecular implementation and physiological roles for histone H3 lysine 4 (H3K4) methylation. Curr Opin Cell Biol. 2008; 20: 341-348.

[7] Pan G, Tian S, Nie J, Yang C, Ruotti V, Wei H, Jonsd ottir GA, Stewart R, Thomson JA. Whole-genome analysis of histone H3 lysine 4 and lysine 27 methylation in human embryonic stem cells. Cell Stem Cell. 2007; 1: 299 -312.

[8] Heintzman ND, Stuart RK, Hon G, Fu Y, Ching CW, Hawkins RD, Barrera LO, Van Calcar S, Qu C, Ching KA, Wang W, Weng Z, Green RD, Crawford GE, Ren B. Distinct and predictive chromatin signatures of tran scriptional promoters and enhancers in the human genome. Nat Genet. 2007; 39: 311-318.

[9] Pasini D, Cloos PA, Walfridsson J, Olsson L, Bukowski JP, Johansen JV, Bak M, Tommerup N, Rappsilber J, Helin K. JARID2 regulates binding of the Polycomb repressive complex 2 to target genes in ES cells. Nature. 2010; 464: $306-310$.

[10] Clouaire T, Webb S, Skene P, Illingworth R, Kerr A, Andrews R, Lee JH, Skalnik D, Bird A. Cfp1 integrates both CpG content and gene activity for accurate H3K4me3 deposition in embryonic stem cells. Genes Dev. 2012; 26: 1714 -1728 .

[11] Jermann P, Hoerner L, Burger L, Schubeler D. Short sequences can efficiently recruit histone H3 lysine 27 trimethylation in the ab sence of enhancer activity and DNA methylation. Proc Natl Acad SciU S A. 2014; 111 : E3415-E3421. 